\title{
Advances in clinical application of quantitative myocardial perfusion imaging
}

\author{
Juhani Knuuti, MD, PhD and Antti Saraste, MD, PhD
}

\section{See related article, pp. 670-680}

Myocardial perfusion imaging (MPI) is well-established and the most widely used noninvasive imaging method for the detection of myocardial ischemia. Numerous studies have shown that the diagnostic accuracy of MPI for the detection of angiographically significant coronary artery disease (CAD) is high and that normal MPI in patients with intermediate-to-high likelihood of CAD predicts a very low rate of cardiac death or nonfatal myocardial infarction (MI). These features make MPI a strong technique to guide selection of patients for revascularization.

The standard interpretation of MPI is based on the assessment of relative myocardial perfusion defects. This method of defining regional reductions in uptake relative to the maximum in the left ventricle myocardium has some limitations in the detection of ischemia. In clinical scenarios, these shortcomings may arise particularly with patients who have multivessel disease. ${ }^{1}$ The relative assessment is based on the assumption that the region with the best perfusion is normal and can be used as a reference. Therefore, global reduction of myocardial perfusion due by diffuse microvascular disease or multivessel disease can lead to false negative result and underestimation of patient's risk. The balanced multivessel disease may be completely missed or sometimes only the territory supplied by the most severe coronary artery stenosis can be identified. This constitutes one of the major shortcomings of standard relative MPI.

It is possible to make quantitative measurements of myocardial blood flow (MBF) and myocardial flow reserve (MFR) with the use of positron-emission

From the Turku PET Centre, University of Turku and Turku University Hospital, Turku, Finland.

Reprint requests: Juhani Knuuti, MD, PhD, Turku PET Centre, University of Turku and Turku University Hospital, Kiinamyllynkatu 4-8, 20520 Turku, Finland; juhani.knuuti@utu.fi.

J Nucl Cardiol 2012;19:643-6.

$1071-3581 / \$ 34.00$

Copyright $(5) 2012$ American Society of Nuclear Cardiology.

doi:10.1007/s12350-012-9530-0 tomography (PET). Owing to high temporal resolution and correction of photon attenuation PET provides accurate delineation of regional tracer kinetics, which are used in combination with validated tracer kinetic models to quantify $\mathrm{MBF}$ in $\mathrm{mL} / \mathrm{minute} / \mathrm{g}$ of tissue. Regional MBF and MFR in response to stressors are reduced in the myocardial regions subtended by the stenosed coronary artery and correlate with the severity of stenosis, measured by quantitative coronary angiography $^{2,3}$ and also with the pressure gradient induced by the stenosis. Thus, in patients with chronic stable angina and no previous history of MI, quantitative assessment of MBF and MFR allows determination of the functional significance of epicardial coronary lesions. However, these measures have not been widely integrated into clinical practice and there is limited data supporting incremental diagnostic utility of flow quantification in patients being assessed for myocardial ischemia.

Recently, there has been growing interest in translation of quantitative flow and flow reserve using PET from mainly research tool to routine clinical practice. This is largely based on the recent growth in the number of hybrid PET and computed tomography (CT) systems, attributable primarily to their wide spread use in clinical oncology. Moreover, the generator produced flow tracer ${ }^{82}$ Rubidium $\left({ }^{82} \mathrm{Rb}\right.$ ) has made perfusion imaging possible at sites without onsite cyclotron. Still, ${ }^{13} \mathrm{~N}$-labeled ammonia $\left({ }^{13} \mathrm{NH}_{3}\right)$ and ${ }^{15} \mathrm{O}$-labeled water $\left(\mathrm{H}_{2}^{15} \mathrm{O}\right)$ are the most often used validated PET tracers for quantification of regional myocardial perfusion. However, they have short half-lives (10 and 2 minutes, respectively) and so they both require on-site cyclotron, which limits their widespread clinical use. Because of the advantage of not requiring an on-site cyclotron, ${ }^{82} \mathrm{Rb}$ is currently the most widely clinically used radionuclide for assessment of myocardial perfusion with PET. ${ }^{82} \mathrm{Rb}$ is a potassium analogue with a physical half-life of 76 seconds enabling high throughput for pharmacological rest-stress studies. Its kinetic properties are similar to those of SPECT tracers and it is well suited for clinical MPI using qualitative or semiquantitative evaluation. For quantification, the limitations of ${ }^{82} \mathrm{Rb}$ are the suboptimal and nonlinear extraction fraction and its higher positron range that can degrade image resolution. However, owing to continued improvements in technology, several groups have now demonstrated that quantitative ${ }^{82} \mathrm{Rb}$ 
PET in humans is feasible, accurate, and reproducible and has been validated against microspheres in animal models of CAD. ${ }^{5-7}$

In the current issue of Journal of Nuclear Cardiology, Ziadi et $\mathrm{al}^{8}$ studied whether assessment of MFR measured with ${ }^{82} \mathrm{Rb}$ PET is an independent predictor of severe obstructive 3-vessel CAD. The authors retrospectively studied 120 patients referred to a dipyridamole PET MPI for evaluation of ischemia who also underwent coronary angiogram within 6 months of the PET study. The patients with and without 3-vessel CAD were compared. Globally reduced MFR $(<2)$ identified patients with severe 3-vessel CAD with sensitivity of $88 \%$. Impaired MFR was an independent predictor of 3-vessel CAD and also the incremental diagnostic value of MFR over the summed stress score (SSS) based on relative analysis was also shown by comparing the adjusted SSS models with and without MFR. The authors concluded that MFR is an independent predictor of 3-vessel CAD and provides added diagnostic value to relative MPI.

The study of Ziadi et al is important in demonstrating that quantitative global MFR provides additional value over the classical defect size in the detection of 3 vessel CAD. This combination works in the way that relative SSS provides data about size of the defect and MFR about the severity of perfusion abnormality in general. Routine list mode acquisition of ${ }^{82} \mathrm{Rb}$ PET scan enables easy combination of flow quantification with the traditional MPI parameters, which facilitates implementation of quantitative analysis. In addition to detection of reversible perfusion defects in areas of ischemia, it is possible to detect areas of fixed defects in areas of MI by conventional analysis. It is notable that other high-risk imaging parameters including ischemic ECG changes, transient ischemic dilatation, and ejection fraction response during stress were often not present in the patients with 3 -vessel CAD in this study. However, it is important to keep in mind that global MFR provides only part of the potential benefit of flow quantification in the assessment of CAD. The ultimate goal would be classification of each myocardial segment or region subtended by the major coronary arteries into normal or abnormal based on MFR followed by regional analysis or calculation of SSS based on those quantitative results.

The possible differences between relative uptake and quantitative blood flow are probable in three different patient groups. ${ }^{1}$ The first group is the patients with 3-vessel disease and balanced, uniform reduction of perfusion that is not detected by relative analysis. The study of Ziadi et al focused on this condition and showed that reduced global MFR can identify such patients. The second major group of discrepant findings between quantitative and relative analyses comprises patients with multivessel disease in whom the presence of CAD is detected using relative uptake analysis, but only the most severe region considered pathological. This is easy to understand, because the region with the best perfusion is considered to represent normal flow in relative analysis, which is, however, not usually the case in patients with multivessel disease. Regional quantitative analysis would allow analysis of each region independently. The third group accounting for discrepancies is presented by patients with high $\mathrm{MBF}$ in the normal range, but still inhomogeneous radiotracer uptake erroneously interpreted as perfusion defects. In this condition, relative analysis would show falsely positive result, whereas the local variances within normal high flow could be separated from regionally compromised perfusion by quantitative analysis.

How often quantitative analysis would lead to reclassification of patients is not known. Obviously, the relative number of patients with multivessel and singlevessel disease as well as impaired microcirculation reflects a particular patient population influenced by the pretest probability of CAD of the patient group. A recent study by Kajander et al prospectively compared the conventional approach of assessing relative radiotracer uptake with quantitative measurement of MBF in 107 patients with intermediate (30\%-70\%) likelihood of CAD based on age, sex, symptom classification, and the result of exercise ECG. ${ }^{9}$ In that study, $\mathrm{H}_{2}^{15} \mathrm{O}$ PET was used to measure absolute regional $\mathrm{MBF}$ among each coronary territory. All patients underwent invasive coronary angiography and measurement of fractional flow reserve (FFR) was added when appropriate. As expected, the interpretation of balanced multivessel disease erroneously as normal by relative analysis was rare $(2 \%$ of patients). However, underestimation of the number of affected vessels was more common (12\% of patients). In that study, the number of patients with inhomogeneous radiotracer uptake erroneously interpreted as perfusion defects at a high perfusion range was relatively large, but this is probably of concern only when tracers with high extraction, such as $\mathrm{H}_{2}^{15} \mathrm{O}$ are used and should not be directly extrapolated to other perfusion tracers used in MPI.

The finding of diffusely reduced MBF and MFR may have important implications for decisions about selecting patients for revascularization procedures as well as prognostic evaluation. It has been shown that in patients evaluated for myocardial ischemia by MPI reduced global MFR was associated with higher rate of adverse cardiovascular events even in the absence of regional ischemia. ${ }^{10,11}$ An interesting subgroup is the patients with normal (or nearly normal) epicardial vessels but with diffusely reduced MBF. The relative perfusion method frequently classifies these patients as 
normal. With quantitative PET, these patients with possible microcirculatory disease often cannot be separated from those with epicardial multivessel disease as also demonstrated by relatively low specificity of reduced MFR for identification of 3-vessel disease in the study of Ziadi et al. ${ }^{8}$ However, it should also be noted that hybrid PET/CT imaging combining perfusion with noninvasive angiography can lead to a correct diagnosis in such patients. ${ }^{12}$

Ongoing research in several areas will provide more information of the diagnostic and prognostic value of quantitative MFR in the assessment of patients with suspected ischemia. Prospective studies assessing the value of both quantitative global and regional MFR in patients without known CAD are under way in larger patient groups than reported so far. In addition to flow reserve, quantitative stress $\mathrm{MBF}$ alone has been proposed to be sufficient for diagnosis of CAD. ${ }^{13}$ The transition from semi-quantitative to quantitative analysis of blood flow involves complex mathematical data modeling depending on accurate determination of tracer input function and myocardial tracer counts over time. Therefore, the quantitative data is also prone to artifacts and especially regional analysis sometimes fails. Although quantitative analysis is used in some, experienced centres, technical developments are required to make it available to more wide spread use. Quantitative analysis of ${ }^{82} \mathrm{Rb}$ scan is demanding, but it is encouraging that a recent study showed very good correlations between different available softwares for the measurement of MBF and MFR with ${ }^{13} \mathrm{NH}_{3}$ PET. ${ }^{14}$ The first clinical study with ${ }^{18} \mathrm{~F}$-labeled PET perfusion tracer, ${ }^{18} \mathrm{~F}$ flurpiridaz was recently reported. ${ }^{15}$ The good kinetic properties and excellent signal-to-noise ratios provided by ${ }^{18} \mathrm{~F}$ flurpiridaz may in part facilitate implementation of quantitative analysis protocols for clinical work-up of patients. Furthermore, due to half-life of 110 minutes, its availability is not limited to centers with on-site cyclotron facilitating its wide spread use in clinical imaging protocols.

\section{CONCLUSIONS}

Quantitative PET measurements of absolute MBF and MFR have potential to improve accuracy of MPI in diagnosis of multivessel CAD as well as definition of the extent and functional importance of stenoses. The study by Ziadi et al shows that flow quantification can be applied in a clinical PET MPI study with a widely available tracer ${ }^{82} \mathrm{Rb}$ and combined with the traditional analysis to easily obtain incremental diagnostic information on the likelihood of extensive CAD. It appears that quantitative analysis of MPI can soon become integral part of clinical MPI.

\section{Acknowledgments}

The authors acknowledge financial support from The Academy of Finland Centre of Excellence on Molecular Imaging in Cardiovascular and Metabolic Research, Helsinki, Finland and Finnish Foundation for Cardiovascular Research.

\section{References}

1. Knuuti J, Kajander S, Maki M, Ukkonen H. Quantification of myocardial blood flow will reform the detection of cad. J Nucl Cardiol 2009;16:497-506.

2. Gould KL, Lipscomb K. Effects of coronary stenoses on coronary flow reserve and resistance. Am J Cardiol 1974;34:48-55.

3. Uren NG, Melin JA, De Bruyne B, Wijns W, Baudhuin T, Camici PG. Relation between myocardial blood flow and the severity of coronary artery stenosis. N Engl J Med 1994;330:1782-8.

4. Di Carli M, Czernin J, Hoh CK, Gerbaudo VH, Brunken RC, Huang SC, et al. Relation among stenosis severity, myocardial blood flow, and flow reserve in patients with coronary artery disease. Circulation 1995;91:1944-51.

5. Lortie M, Beanlands RS, Yoshinaga K, Klein R, Dasilva JN, DeKemp RA. Quantification of myocardial blood flow with 82-Rb dynamic PET imaging. Eur J Nucl Med Mol Imaging 2007;34: 1765-74.

6. Anagnostopoulos C, Almonacid A, El Fakhri G, Curillova Z, Sitek A, Roughton M. Quantitative relationship between coronary vasodilator reserve assessed by $82-\mathrm{Rb}$ PET imaging and coronary artery stenosis severity. Eur J Nucl Med Mol Imaging 2008;35: 1593-601.

7. Lautamäki R, George RT, Kitagawa K, Higuchi T, Merrill J, Voicu C, et al. Rubidium-82 PET-CT for quantitative assessment of myocardial blood flow: Validation in a canine model of coronary artery stenosis. Eur J Nucl Med Mol Imaging 2009;36: 576-86.

8. Ziadi MC, deKemp RA, Williams K, Guo A, Renaud JM, Chow BJW, et al. Does quantification of myocardial flow reserve using rubidium-82 positron emission tomography facilitate detection of multivessel coronary artery disease? J Nucl Cardiol 2012. doi: 10.1007/s12350-011-9506-5

9. Kajander SA, Joutsiniemi E, Saraste M, Pietilä M, Ukkonen H, Saraste A, et al. Clinical value of absolute quantification of myocardial perfusion with (15)O-water in coronary artery disease. Circ Cardiovasc Imaging 2011;4:678-84.

10. Herzog BA, Husmann L, Valenta I, Gaemperli O, Siegrist PT, Tay FM, et al. Long-term prognostic value of $13 \mathrm{~N}$-ammonia myocardial perfusion positron emission tomography added value of coronary flow reserve. J Am Coll Cardiol 2009;54:150-6.

11. Ziadi MC, Dekemp RA, Williams KA, Guo A, Chow BJ, Renaud $\mathrm{JM}$, et al. Impaired myocardial flow reserve on rubidium-82 positron emission tomography imaging predicts adverse outcomes in patients assessed for myocardial ischemia. J Am Coll Cardiol 2011;58:740-8.

12. Kajander S, Joutsiniemi E, Saraste M, Pietilä M, Ukkonen H, Saraste A, et al. Diagnostic performance of hybrid CT coronary angiography and positron emission tomography for coronary artery disease. Circulation 2010;122:603-13.

13. Hajjiri MM, Leavitt MB, Zheng H, Spooner AE, Fischman AJ, Gewirtz H. Comparison of positron emission tomography measurement of adenosine-stimulated absolute myocardial blood flow versus relative myocardial tracer content for physiological assessment of coronary artery stenosis severity and location. JACC Cardiovasc Imaging 2009;2:751-8 
14. Slomka PJ, Alexanderson E, Jácome R, Jiménez M, Romero E, Meave A et al. Comparison of clinical tools for measurements of regional stress and rest myocardial blood flow assessed with 13N-ammonia PET/CT. J Nucl Med 2012;53(2): 171-81.
15. Maddahi J, Czernin J, Lazewatsky J, Huang SC, Dahlbom M, Schelbert H, et al. Phase I, first-in-human study of BMS747158, a novel 18F-labeled tracer for myocardial perfusion PET: Dosimetry, biodistribution, safety, and imaging characteristics after a single injection at rest. J Nucl Med 2011;52:1490-8. 